\title{
Oblique incidence of semi-guided waves on rectangular slab waveguide discontinuities: A vectorial QUEP solver
}

\author{
Manfred Hammer ${ }^{1}, 2$, \\ ${ }^{1} \mathrm{MESA}^{+}$Institute for Nanotechnology, University of Twente, Enschede, The Netherlands \\ ${ }^{2}$ Theoretical Electrical Engineering, University of Paderborn, Germany
}

\begin{abstract}
The incidence of thin-film-guided, in-plane unguided waves at oblique angles on straight discontinuities of dielectric slab waveguides, an early problem of integrated optics, is being reconsidered. The 3-D frequency domain Maxwell equations reduce to a parametrized inhomogeneous vectorial problem on a 2 -D computational domain, with transparent-influx boundary conditions. We propose a rigorous vectorial solver based on simultaneous expansions into polarized local slab eigenmodes along the two orthogonal cross section coordinates (quadridirectional eigenmode propagation QUEP). The quasi-analytical scheme is applicable to configurations with - in principle — arbitrary cross section geometries. Examples for a high-contrast facet of an asymmetric slab waveguide, for the lateral excitation of a channel waveguide, and for a step discontinuity between slab waveguides of different thicknesses are discussed.
\end{abstract}

Keywords: integrated optics, slab waveguide discontinuities, thin-film transitions, waveguide facets, numerical/analytical modeling, vectorial eigenmode expansion.

PACS codes: 42.82.-m 42.82. Bq 42.82.Et 42.82.Gw 42.15.-i

\section{Introduction}

The effects of transitions between regions with different layering on thin-film guided, in-plane unguided light form the basis for a series of classical integrated optical components [1, 2]. Concepts have been discussed for lenses [3, 4], mirrors [5], prisms [6], but also for complex lens-systems [7], or entire spectrometers [8]. When looked at from a direction perpendicular to the slab plane ("top view"), the relevant interfaces are either straight, or merely slightly curved, permitting a description of the in-plane wave propagation in terms of geometrical optics. Hence we take a closer look at what happens to vertically guided, laterally plane waves at straight interfaces, facets, or transition regions with other cross section shapes. Figure 1 illustrates the configurations considered as examples in this paper.
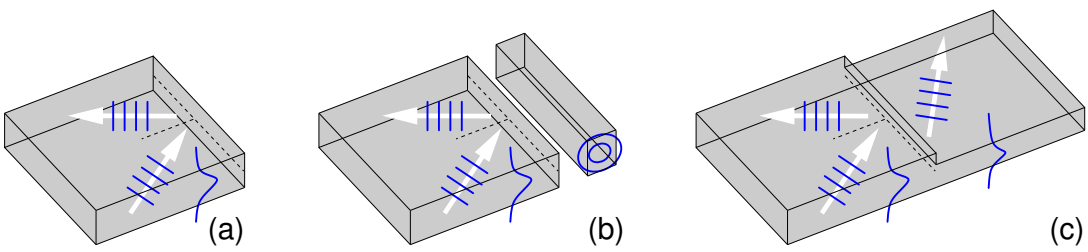

Figure 1: Oblique incidence of vertically guided, laterally unguided plane waves on a slab facet (a), a facet coupled to a strip waveguide (b), and a step discontinuity between regions with different core thicknesses (c).

While standard scalar TE/TM Helmholtz equations apply for perpendicular incidence, for non-normal incidence one is led to a vectorial problem [9] that is formally identical to that for the modes of 3-D channel waveguides [10]. Here, however, it needs to be solved as a parametrized, inhomogeneous system on a 2-D computational window with transparent-influx boundary conditions, with a right-hand-side given by the incoming wave.

Starting point for a semi-analytical treatment is the observation that the structures under consideration can be separated into segments with locally constant permittivity along the ("horizontal") axis perpendicular to the waveguide discontinuities, and with piecewise constant permittivity in the second ("vertical") direction normal to the slab plane. With the TE/TM modes supported by multilayer slab waveguide configurations, complete sets of local solutions for the separate segments are readily at hand. It remains to "rotate" the vector fields associated with these slab modes to account for wave propagation at angles as prescribed by the incident field, and then to properly match the individual expansions at the segment interfaces. Respective approaches have

* University of Paderborn, FG Theoretical Electrical Engineering

Warburger Str. 100, 33098 Paderborn, Germany

Phone: ++49(0)5251/60-3560

Fax: $++49(0) 5251 / 60-3524$

E-mail:m.hammer@utwente.nl 
been described already more than two decades ago [11, 12], but have apparently hardly (not ?) been continued. This might be due to problems with convergence of the bidirectional algorithms in case of basis mode sets discretized by "hard" boundary conditions, and / or with the limits of standard computing power.

As a step beyond these older bidirectional techniques [11, 12], and also beyond our previous study in the framework of the scalar approximation [9], we here report on a dedicated vectorial solver for - in principle - arbitrary rectangular cross section geometries, based on simultaneous expansions into slab modes along two orthogonal coordinate axes. The scheme constitutes a vectorial extension of the scalar approach of Ref. [13] (quadridirectional eigenmode propagation, QUEP).

The description of the formal problem in Section 2 includes brief accounts of general aspects of the solutions. Although some concepts have been reported in Refs. [11, 12], for reasons of self-consistency we deem it appropriate to include respective paragraphs in the present paper. This concerns the vectorial properties of "rotated" slab modes, occurrence of critical angles, remarks on reciprocal behaviour, and approximate expressions for what happens to localized beams at the discontinuities, in Sections 2.1 2.4. Section 3 outlines the extended QUEP scheme, basically referring to the original description [13] of the algorithm, with emphasis on the modifications necessary for the vectorial implementation. The examples in Section 4 intend to illustrate the solutions, to provide some numerical assessment, and to connect with the previous scalar results of Ref. [9].

\section{Oblique wave incidence on slab waveguide discontinuities}

Figure 2 introduces a facet configuration along which the solver concepts will be described. The vertical $x$ and horizontal $z$-axes span the cross section plane of the configuration, the entire structure (not the solutions) is supposed to be constant along the $y$-axis.
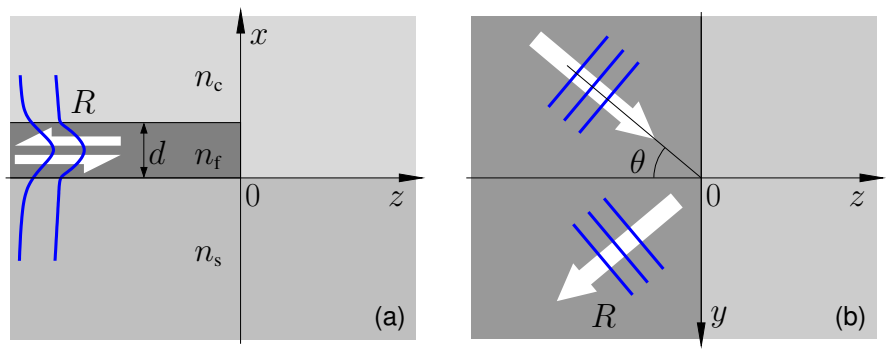

Figure 2: Facet of a slab waveguide, incidence of vertically $(x)$ guided, laterally $(y, z)$ unguided plane waves at angle $\theta$; cross-section view (a) and top view (b). Parameters (cf. Section 4.1): refractive indices $n_{\mathrm{c}}=1.0$ (cover), $n_{f}=2.0$ (film), $n_{\mathrm{s}}=1.5$ (substrate), core thickness $d=0.5 \mu \mathrm{m}$, vacuum wavelength $\lambda=1.55 \mu \mathrm{m}$.

The problem is governed by the homogeneous Maxwell equations in the frequency domain, for linear, dielectric, and nonmagnetic media,

$$
\operatorname{curl} \tilde{\boldsymbol{E}}=-\mathrm{i} \omega \mu_{0} \tilde{\boldsymbol{H}}, \quad \operatorname{curl} \tilde{\boldsymbol{H}}=\mathrm{i} \omega \epsilon \epsilon_{0} \tilde{\boldsymbol{E}}
$$

for electric and magnetic fields $\tilde{\boldsymbol{E}}, \tilde{\boldsymbol{H}}$, oscillating $\sim \exp (\mathrm{i} \omega t)$ in time with angular frequency $\omega=k \mathrm{c}=2 \pi \mathrm{c} / \lambda$, for wavenumber $k$, wavelength $\lambda$, speed of light $\mathrm{c}$, permittivity $\epsilon_{0}$, and permeability $\mu_{0}$ (all vacuum values). Structural information is given by the rectangular, piecewise constant relative permittivity $\epsilon=n^{2}$, or refractive index $n$, both functions of the cross section coordinates $x, z$ only: $\partial_{y} \epsilon=0, \partial_{y} n=0$.

For normal wave incidence one would here restrict the formalism to fields that are constant along the $y$-direction as well; this leads to the familiar scalar TE / TM Helmholtz problems. Instead, to accommodate for the incoming wave at non-normal angles, one introduces a respective uniform harmonic $y$-dependence of all optical fields:

$$
\left(\begin{array}{c}
\tilde{\boldsymbol{E}} \\
\tilde{\boldsymbol{H}}
\end{array}\right)(x, y, z)=\left(\begin{array}{c}
\boldsymbol{E} \\
\boldsymbol{H}
\end{array}\right)(x, z) \mathrm{e}^{-\mathrm{i} k_{y} y}
$$

Here the lateral wavenumber $k_{y}$ is a given parameter, determined by the incoming field.

Note that Eq. (2) is formally identical to the standard ansatz for the modes of channel waveguides with 2-D confinement [14, 10]. For modal analysis, the present parameter $k_{y}$ would play the role of the unknown propagation constant, and the respective - then homogeneous - equations would be considered as an eigenvalue problem, to be solved on a computational domain with boundary conditions of vanishing field components, or 
of outgoing fields only. Here, on the contrary, the incoming wave leads to inhomogeneous equations, transparent boundary conditions are essential to account for any radiative losses, and the influx has to be accommodated by the boundary conditions [9].

\subsection{Vectorial slab modes}

For the facet example in Figure 2, the regions $z<0$ and $z>0$ are distinguished by the property of $y$ - and $z$-constant permittivity: $\partial_{y} \epsilon=0, \partial_{z} \epsilon=0$. As can be shown by restricting Eqs. (1), using (2), local solutions in these regions are given by pairs $\psi, \beta$ of eigenfunctions and -values, solutions of the TE- and TM slab mode equations associated with the local profile $\epsilon=\epsilon(x)$. These are fields of the form

(TE) $\quad \partial_{x}^{2} \psi+\left(k^{2} \epsilon-\beta^{2}\right) \psi=0$,

$$
\boldsymbol{E}(x, z)=\left(\begin{array}{c}
0 \\
k_{z} \psi(x) / \beta^{2} \\
-k_{y} \psi(x) / \beta^{2}
\end{array}\right) \mathrm{e}^{-\mathrm{i} k_{z} z}, \quad \boldsymbol{H}(x, z)=\frac{1}{\omega \mu_{0}}\left(\begin{array}{c}
-\psi(x) \\
\mathrm{i} k_{y} \partial_{x} \psi(x) / \beta^{2} \\
\mathrm{i} k_{z} \partial_{x} \psi(x) / \beta^{2}
\end{array}\right) \mathrm{e}^{-\mathrm{i} k_{z} z}
$$

and

$$
\begin{aligned}
& \text { (TM) } \quad \epsilon \partial_{x} \frac{1}{\epsilon} \partial_{x} \psi+\left(k^{2} \epsilon-\beta^{2}\right) \psi=0, \\
& \boldsymbol{E}(x, z)=\frac{1}{\omega \epsilon_{0} \epsilon}\left(\begin{array}{c}
\psi(x) \\
-\mathrm{i} k_{y} \partial_{x} \psi(x) / \beta^{2} \\
-\mathrm{i} k_{z} \partial_{x} \psi(x) / \beta^{2}
\end{array}\right) \mathrm{e}^{-\mathrm{i} k_{z} z}, \quad \boldsymbol{H}(x, z)=\left(\begin{array}{c}
0 \\
k_{z} \psi(x) / \beta^{2} \\
-k_{y} \psi(x) / \beta^{2}
\end{array}\right) \mathrm{e}^{-\mathrm{i} k_{z} z},
\end{aligned}
$$

where in both cases the relation $\beta^{2}=k_{y}^{2}+k_{z}^{2}$ between the directional wavenumbers and the slab mode propagation constant $\beta$ determines the wavenumber $k_{z}$ of the respective mode in the cross section problem on the $x$ - $z$-plane.

Extension of Eqs. (3), (5) by artificial boundary conditions on a finite $x$-interval leads to a discretization of the slab mode spectra for further numerical processing. Boundary conditions of both Dirichlet-type $\psi=0$ and of Neumann-type $\partial_{x} \psi=0$ will play a role in the scheme introduced in Section 3

Analogous steps can be followed for regions where the permittivity is constant along $y$ and $x$. By exchanging the roles of $x$ and $z$ in the derivations leading to Eqs. (3)-(6), one readily writes expressions for slab modes that travel along $\pm x$ with wavenumbers $k_{x}$, and with $z$-dependent profiles.

Interest is in what happens for influx of a polarized guided mode of the form of Eq. (4) or Eq. (6), with propagation constant $\beta_{\text {in }}=k N_{\text {in }}$ and effective mode index $N_{\text {in }}$, coming in at an angle $\theta$. According to Figure 2 the uniform lateral wavenumber $k_{y}$ (real in the present context) has to be chosen as

$$
k_{y}(\theta)=k N_{\text {in }} \sin \theta \text {. }
$$

Once $k_{y}$ is fixed by the choice of the input field and angle of incidence, the $z$-wavenumbers for all other local modes are determined by the relation

$$
k_{z}(\theta)= \pm \sqrt{\beta^{2}-k_{y}^{2}(\theta)},
$$

where $\beta$ results from the respective modal eigenvalue problems, and the sign distinguishes propagation directions. Consequently, when looking at variations of the angle of incidence, the propagation characteristics can change between $z$-propagating $\left(\beta^{2}-k_{y}^{2}>0\right.$, real $\left.k_{z}\right)$ and $z$-evanescent, individually for all modes in the discretized spectra. Note that this concerns horizontally as well as vertically traveling fields. 


\subsection{Critical angles}

Arguing in line with the computational setting of Section 3 , for a limiting case of large extension of the interior window, all outgoing fields can be viewed as being composed of local eigenmodes of the form (4), (6), either horizontally or vertically traveling, and with the sign of $k_{z}$, or $k_{x}$, respectively, chosen such that the fields are either outwards propagating, or, in case of evanescent modes, the fields are outwards exponentially decaying. Note that this encompasses all modes in the complete expansions for arbitrary, but outwards constant, external permittivity profiles, irrespective of whether actual slabs with truly guided modes are present or not. Also these fields share the uniform exponential $y$-dependence. For an outgoing, originally propagating mode with propagation constant $\beta_{\text {out }}=k N_{\text {out }}, \beta_{\text {out }}^{2}>0$, this defines an outgoing angle $\theta_{\text {out }}$ through the relations $k_{y}=$ $k N_{\text {out }} \sin \theta_{\text {out }}$, or

$$
N_{\text {out }} \sin \theta_{\text {out }}=N_{\text {in }} \sin \theta .
$$

Eq. (9) may be viewed as a generalized form of Snell's law, here applicable to all outgoing (reflected, transmitted, up- or downwards scattered, guided or nonguided, of both polarizations) propagating modes. Consequently, in cases where multiple of these with different effective mode indices exist, these outgoing waves are observed all under different angles.

It can be shown that the statements concerning power transport and mode orthogonality of sets of "ordinary" slab eigenmodes (in the present context: fields (4), (6) for $k_{y}=0$ ), as summarized e.g. in Ref. [15], remain valid for the vectorial situation. Consequently, for some given input angle $\theta$, only those modes can carry power away from the discontinuity, for which $k_{z}$ according to Eq. (8) turns out to be real,

Frequently, as in the examples of Section 4 one considers a dependence of power transfer (modal reflectance, transmittance, radiative losses) on incidence angles. When increasing this angle, formerly $z$-propagating modes can convert to $z$-evanescent, and thus cease to contribute to the outgoing power. Consider an outgoing mode with effective index $N_{\text {out }} \leq N_{\text {crit }}$ below some critical value $N_{\text {crit }}$, translated to a critical angle $\theta_{\text {crit }}$ by $\sin \theta_{\text {crit }}=N_{\text {crit }} / N_{\text {in }}$. For all angles of incidence $\theta \geq \theta_{\text {crit }}$ one then reasons that $\sin \theta_{\text {out }}=N_{\text {in }} \sin \theta / N_{\text {out }} \geq$ $N_{\text {in }} \sin \theta_{\text {crit }} / N_{\text {out }} \geq N_{\text {in }} \sin \theta_{\text {crit }} / N_{\text {crit }}=1$, and consequently the respective mode must be evanescent, not contributing to the outgoing power in any form.

Merely on the basis of these quite general considerations one can already establish some values for critical angles at which the respective power transmission curves might exhibit more or less pronounced kinks. For the configuration and parameters as introduced in Figure 2, relevant for Sections 4.1, 4.2, one has:

- Fields that can propagate in the cover region are characterized by effective mode indices $N \leq n_{\mathrm{c}}$. Hence, for incidence angles $\theta \geq \theta_{\mathrm{c}}$ with $\sin \theta_{\mathrm{c}}=n_{\mathrm{c}} / N_{\mathrm{in}}$, there is no power radiated (lost) into the cover region.

- Fields that can propagate in the substrate region are characterized by effective mode indices $N \leq n_{\mathrm{s}}$. For incidence angles $\theta \geq \theta_{\mathrm{s}}$ with $\sin \theta_{\mathrm{s}}=n_{\mathrm{s}} / N_{\text {in }}$, there is no power radiated (lost) into either the cover or substrate regions; all incident power is being carried away by the reflected guided TE and TM modes.

- For incidence of the fundamental TE mode, with effective mode index $N_{\mathrm{TE} 0}>N_{\mathrm{TM} 0}$ larger than that of the fundamental TM mode, and for incidence at angles $\theta \geq \theta_{\mathrm{m}}$ with $\sin \theta_{\mathrm{m}}=N_{\mathrm{TM} 0} / N_{\mathrm{TE} 0}$, the reflected $\mathrm{TE}_{0}$ mode carries the entire incident power, without any radiative losses or polarization conversion.

Inequalities $N_{\mathrm{TE} 0}>N_{\mathrm{TM} 0}>n_{\mathrm{s}}>n_{\mathrm{c}}$ apply. Note that the "suppressed", evanescent modal fields contribute well to the total field in the region around the discontinuity, merely without transporting power away.

\subsection{Reciprocal configurations}

The notion of reciprocal behaviour [10, 12] concerns waves characterized by opposite wavevectors, here the $y$ and $z$ components, i.e. configurations with equal (absolute) values $k_{y}=N_{\text {in }} \sin \theta$. Since $N_{\text {in }}$ differs for the different input modes (polarizations), output amplitudes for respectively different angles of incidence should be compared when looking at reciprocity properties.

Specifically for the facet of Figure 2, incidence of the fundamental TE and TM modes will be considered. One expects equal levels of modal reflectance $R_{\mathrm{TE} 0 \rightarrow \mathrm{TM} 0}$ for $\mathrm{TE}_{0}$-input at angles $\theta_{\mathrm{E}}$ and $R_{\mathrm{TM} 0 \rightarrow \mathrm{TE} 0}$ for $\mathrm{TM}_{0}$-input 
at angles $\theta_{\mathrm{M}}$, if the angles are related by $k_{y}=k N_{\mathrm{TE} 0} \sin \theta_{\mathrm{E}}=k N_{\mathrm{TM} 0} \sin \theta_{\mathrm{M}}$. The abscissae in the panels of Figures 6 , 8, and 10 related to TM input have been adjusted accordingly. Respective arguments apply to all pairs of different and orthogonal propagating input / output modes, if present for a particular configuration [12].

\subsection{Beam displacement}

Continuing the discussion from [9], we briefly look at bundles of solutions (2), summed over a narrow range of lateral wavenumbers $k_{y}=k N_{\text {in }} \sin \theta$, or of angles of incidence $\theta$, respectively, around some principal value $\theta_{0}$. These can describe the effects of the discontinuity on vertically guided, in-plane wide, non-guided beams of light [16, 14].

If, for fixed input field, one focuses on one guided outgoing mode, with a relative amplitude whose absolute value is approximately constant over the relevant wavenumber range, the reasoning of Ref. [9], there discussed for total reflection of one particular mode, can be applied. One obtains an expression

$$
\Delta=\left.\frac{1}{k N_{\text {in }} \cos \theta_{0}} \frac{\mathrm{d} \phi}{\mathrm{d} \theta}\right|_{\theta_{0}}
$$

for the lateral displacement $\Delta$, the Goos-Hänchen-shift [14, 9], that the outgoing wave bundle exhibits relative to the incoming beam (cf. Figure 3). Here $\phi(\theta)$ is the phase of the relative amplitude of the outgoing mode, determined for a uniform reference coordinate (a position $z$ for horizontal, $x$ for vertically traveling outgoing modes).

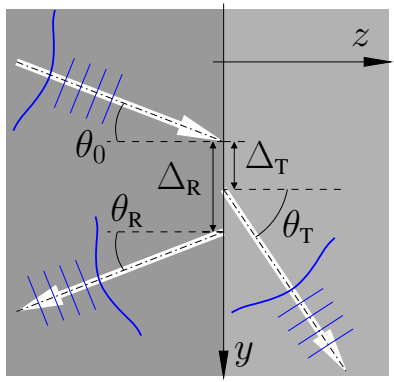

Figure 3: Incidence of a semi-guided wave bundle on a discontinuity, here with reference coordinate $z=0$, at angle $\theta_{0}$. The centers of reflected and transmitted beams associated with different outgoing modes are shifted by distances $\Delta_{R}, \Delta_{T}$ relative to the input bundle. Depending on the configuration in question, positive and negative shifts can possibly occur [17]. The reasoning applies as well for extended and / or composite transitions, e.g. for the strip configurations of Section 4.2, or the tapered structures of [9].

For the examples in Section 4 , the given values for $\Delta$ rely on the phase information extracted from the numerical solver, with a finite difference approximation of the $\theta$-derivative. Note that the shift (10) will be different for different outgoing modes, and that the reasoning can be applied to any reflected, transmitted, up- or downwards traveling guided mode, whatever applies to the configuration in question.

\section{Vectorial quadridirectional eigenmode propagation}

Just as its scalar counterpart [13], the vectorial variant vQUEP operates on a computational setting as introduced in Figure 4, applicable to configurations with rectangular, piecewise constant permittivity. The computational domain consists of the rectangular interior, together with the half-infinite exterior regions. Artificial boundary conditions of Dirichlet- or Neumann-type are being enforced, via the local basis sets, on the boundaries (bold lines) that restrict the exterior regions. One could thus view the computed fields either as solutions for the unlimited cross-shaped domain, or alternatively as approximations to the solutions of the true open problem, restricted to the rectangular window, with boundaries that are transparent for in- and outflux of guided and nonguided waves, but that might be disturbed by numerical artifacts originating from the four corner points of the interior rectangle. The computational domain needs to be selected accordingly.

Starting point for the algorithm is the computation of the incoming field, here one of the propagating, polarized slab modes supported by the permittivity $\epsilon\left(x, z<z_{0}\right)$, with effective modal index $N_{\text {in }}$. Specification of the angle of incidence $\theta$ fixes $k_{y}$ according to Eq. (7). Via the slab mode bases this parameter enters all subsequent steps of the algorithm. Multimode influx, or simultaneous wave influx from more that one direction, can be handled in a single computation in principle [13] (partly obsolete due to the linearity of the problem), provided 


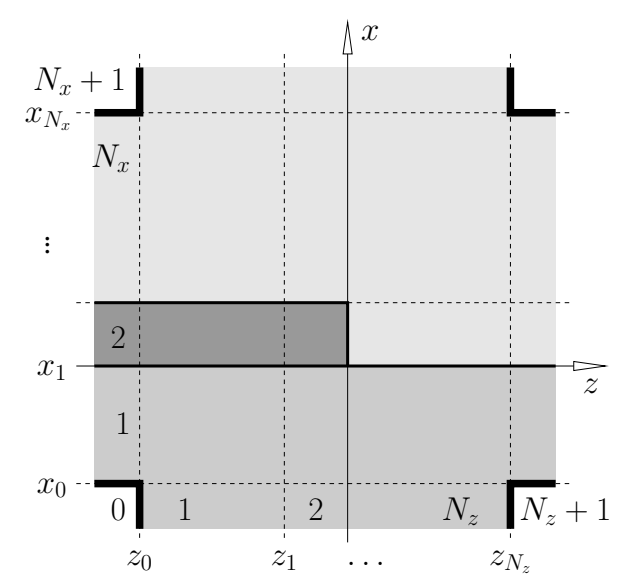

Figure 4: Cross-shaped computational domain for the vQUEP scheme, consisting of the inner computational window $(x, z) \in\left[x_{0}, x_{N_{x}}\right] \times$ $\left[z_{0}, z_{N_{z}}\right]$ and directionally half infinite external regions. The interior rectangle is being divided into $N_{z}$ inner slices with local $z$-constant permittivity, by interfaces at positions $z_{j}$, and into $N_{x}$ inner layers with local $x$ constant permittivity, by interfaces at positions $x_{j}$. The ( $v$-)QUEP scheme (cf. Ref. [13], eqns. (20)-(22) and onwards), and its implementation [18] formally require $N_{z} \geq 3$ and $N_{x} \geq 3$. Hence, an additional artificial interface has been introduced at an arbitrary position $z_{1}$ with $z_{0}<z_{1}<z_{2}$, here for the facet example of Figure 2.

that all incoming fields share the common harmonic dependence on $y$ as in Eq. (2), with possibly different angles of incidence.

The next step is to establish separate expansion bases for each slice / layer, consisting of local slab modes in the form of Eqs. (3)-(6). To account for the potentially hybrid fields due to polarization coupling at the discontinuities, modes of TE- and TM-polarization are included for each region. Their principal functions $\psi$ are restricted by artificial boundary conditions at $x=x_{0}, x_{N_{x}}$ (slices) or at $z=z_{0}, z_{N_{z}}$ (layers). The type of boundary condition needs to ensure that the behaviour at the boundaries is "compatible" for all vectorial TE and TM basis fields in the expansion for the same slice / layer, i.e. that identical nonzero electromagnetic components of all modes in a local set satisfy the same type of condition at the boundary. According to the expressions (4), (6) for the components of the vectorial fields, for the expansions on all slices we've thus chosen boundary conditions of D-type $\psi=0$ for TE, and of $\mathrm{N}$-type $\partial \psi=0$ for TM modes. Corner artifacts showed to be the less pronounced, if, conversely, conditions of N-type for TE and of D-type for TM modes are applied for the expansions on all layers. Further, per polarization (TE / TM), the mode sets are restricted to a finite number of terms $M_{x}$ on each slice and $M_{z}$ on each layer, selected in accordance with the $x$ - and $z$-extensions of the computational window. The resulting expansions of the total field into local basis sets coincide formally with the expressions in Ref. [13].

One accordingly proceeds with the algorithmic scheme as detailed in Ref. [13]. For each vertical and horizontal interface, the tangential components of the total local fields on both sides are formally equated, the equations then projected in turn onto each of the directional variants of the basis modes on both sides, using the vectorial products [15] as applied already Ref. [13]. These bidirectional mode overlaps establish an inhomogeneous linear system of equations for the unknown coefficients of the basis modes in the local expansions, with a right-hand side that is constituted by the given amplitude of the incoming field.

(a)
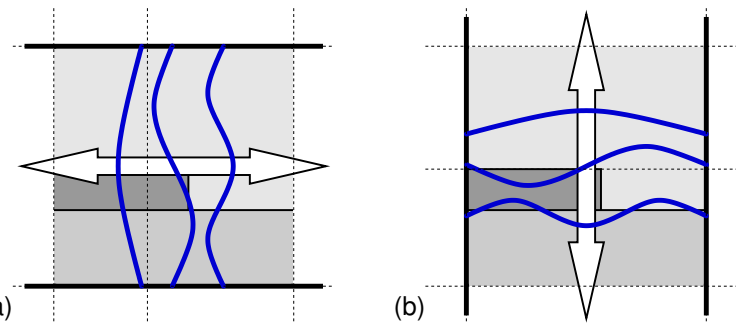

(c)

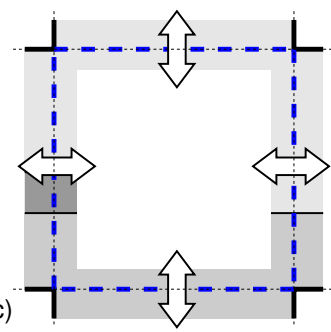

Figure 5: vQUEP, algebraic solution procedure, schematically: horizontal $v B E P$ over the inner slices (a), vertical vBEP over the inner layers (b), and connection of interior and exterior expansions through outermost interfaces (c).

The algebraic solution procedure [13] then consists basically of three phases, as sketched in Figure 5] Due to local exact solutions on the individual slices and layers, the interior horizontal and vertical expansions decouple. The respective parts of the linear systems can be treated independently by a conventional algorithm for bidirectional eigenmode propagation, applied here to the basis sets of vectorial slab modes ("vectorial bidirectional eigenmode propagation", vBEP). Horizontal and vertical vBEP steps are carried out that extend over the domains of the inner slices and inner layers, respectively. In a last step, cross-overlaps at the outermost interfaces relate the partial inner solutions and the field expansions on the external regions. Since vectorial modes propagating in four principal directions $\pm x, \pm z$ play a role, this scheme will be named "vectorial quadridirectional eigenmode propagation" (vQUEP). 


\section{Examples}

The vQUEP scheme as outlined before has been implemented by modifying and extending the existing C++ code [18] for the scalar QUEP. As expected, for perpendicular incidence $\theta=0$ of a polarized guided mode, the vectorial program reproduces the results of the scalar TE- or TM-scheme, with numerically zero amplitudes for the respective other polarization.

Principal computational parameters are the computational window, the inner rectangle as introduced in Figure 4 and the numbers of modal expansion terms $M_{x}, M_{z}$ (terms per slice/layer, per basis mode polarization). These were generally chosen in such a way that, on the scale of the plots as given, the results appear reasonably converged with respect to changes in these parameters (checked at least occasionally). Larger windows, with a correspondingly higher number of spectral terms, are required for configurations with pronounced radiative losses, as e.g. the range of intermediate angles of incidence for the facet example (cf. Figure 6), to suppress the disturbance by corner artifacts. Much smaller windows, and lower numbers of basis elements, are sufficient in case of configurations without radiative losses, e.g. the regime of total reflection around the channel mode resonances for the strip example (Figure 10). In all cases the window needs to cover, with some ample margin, at least the guided slab modes that play a role. Further the observations of Ref. [13] on the computational setting apply; more details and a few examples for computational parameters are given for the comparison of vBEP and vQUEP simulations (Figure 8).

\subsection{Reflection at a waveguide facet}

Figure 6 summarizes results for the configuration of Figure 2. Note the different horizontal scales adopted for the plots with TE excitation (top) and TM input (bottom), introduced to place reciprocal configurations with the same wavenumber $k_{y}$ directly on top of each other.
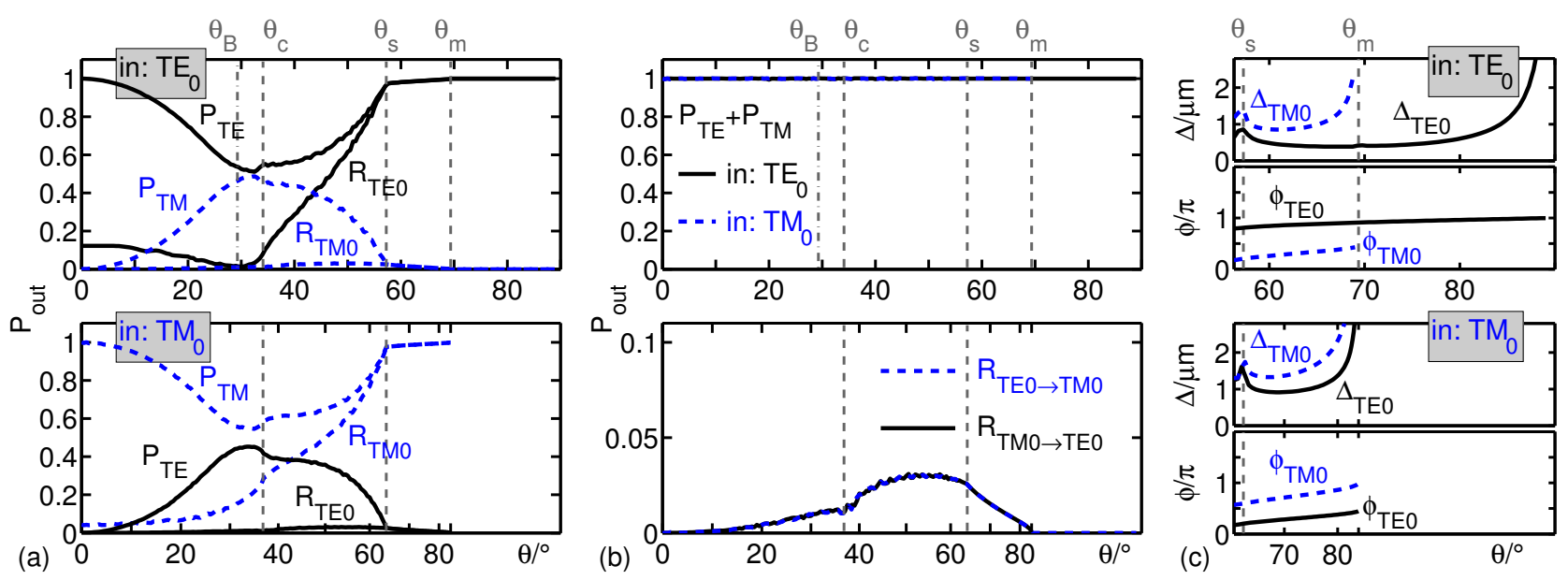

Figure 6: Results for a waveguide facet with the parameters as in Figure 2, scans over the angle of incidence $\theta$. Power distribution (a): relative guided power $R_{T E O}, R_{T M O}$ reflected to the $T E_{0}$ and $T M_{0}$ modes, and total relative outgoing power $P_{T E}, P_{T M}$ carried by $T E$ - and $T M$-modes, respectively, for incidence of $T E_{0}$ - (top) and $T M_{0}$-waves (bottom). Consistency (b): total outgoing power observed, for unit input (top), and polarization-converted reflectance (bottom); note that the upper abscissa applies for TE-input, the lower for TM input. Beam shift properties (c): phase change upon reflection $\phi$ and associated Goos-Hänchen shift $\Delta$.

Part (a) shows the power transfer to guided reflected modes, and the total outgoing power carried away by TEand TM-polarized fields across the four boundaries of the computational window. The guided wave reflectance varies from low initial levels at normal incidence to (nearly) full reflection for grazing incidence. While only low levels of power are converted to the guided reflected mode of the respective other polarization (cf. the lower panel of part (b)), one observes strong conversion for the non-guided parts of the optical fields.

The curves exhibit more or less pronounced kinks at the critical angles as introduced in Section 2.2. Radiative losses to the cover region are suppressed for $\theta>\theta_{\mathrm{c}}, \theta_{\mathrm{c}}=34.11^{\circ}$ for TE, and $\theta_{\mathrm{c}}=36.81^{\circ}$ for TM input. For incidence at angles $\theta>\theta_{\mathrm{s}}$ with $\theta_{\mathrm{s}}=57.28^{\circ}(\mathrm{TE})$ and $\theta_{\mathrm{s}}=63.99^{\circ}(\mathrm{TM})$, all radiative losses are suppressed, merely the guided TE- and TM reflectance remains. For $\theta>\theta_{\mathrm{m}}=69.40^{\circ}$, the incoming fundamental TE wave is fully reflected (due to $N_{\mathrm{TE} 0}>N_{\mathrm{TM} 0}$, this does not happen for TM polarization). 
In the case of bulk optics, the reflectance of p-polarized waves at a dielectric interface between media with refractive indices $n_{1}$ (input side) and $n_{2}$ vanishes for wave incidence at angle $\theta_{\mathrm{B}}$ with $\tan \theta_{\mathrm{B}}=n_{2} / n_{1}$. Reasoning, for the facet, heuristically from a top-view viewpoint, where TE relates to p-polarization for the $y$-z-plane of incidence, one might expect a low modal reflectance for the $\mathrm{TE}_{0}$ mode at wave incidence around the "quasiBrewster angle" $\theta_{\mathrm{B}}$ [12] with $\tan \theta_{\mathrm{B}}=n_{\mathrm{c}} / N_{\mathrm{TE} 0}$. One indeed finds a low $\mathrm{TE}_{0}$-reflectance at the respective value of $\theta_{\mathrm{B}}=29.3^{\circ}$ (where other choices, e.g. $n_{1}=n_{\mathrm{f}}$ and / or $n_{2}=n_{\mathrm{s}}$ would have been plausible as well).

Part (b) of Figure 6 means to provide some consistency check for the simulations. The incoming unit optical power exits the computational window across its four boundaries, carried by outgoing propagating, guided and nonguided modes of both polarizations. As evidenced by the curves, the power is reasonably well conserved. The check of this property provides a convenient way to assess the choice of the spectral density. A toolow number of terms in the expansions usually leads to a pronounced violation of the power balance. For all data in this paper, the total relative outgoing power lies within the interval $[0.995,1.0025]$ (cf. the respective remarks in Ref. [13]). Results can not be trusted to a level better than that accuracy. The lower panel of Figure 6(b) compares the levels of relative guided power conversion caused by incoming fundamental modes of both polarization, for reciprocal configurations. As it should be, the curves coincide nicely on the already magnified scale of the plot.

The last part (c) of Figure 6 shows the beam displacement properties of the facet, restricted to the regime of large angles of incidence with total reflection of guided power. The largest displacements $\Delta$ occur for grazing incidence. Different shifts are predicted for the mayor reflected beam with unchanged polarization, and the minor, polarization converted bundle, if existent.

The simulations provide full vectorial fields for the (quasi-) 3-D configurations; Figure 7 tries to give an impression. These are fields of two reciprocal configurations at angles of incidence close to the quasi-Brewster configuration for TE input.


Figure 7: Absolute values of electromagnetic field components for the facet of Figure 2, for angles of incidence $\theta=30^{\circ}$ for $T E_{0}$ excitation (a), and $\theta=32.3^{\circ}$ for $T M_{0}$ input (b). The color scales have been adjusted such that levels are comparable in each row of panels.

One observes pronounced power loss (for TE polarization nearly all power is radiated), with pronounced wave bundles escaping mainly into the substrate, but also partly into the cover region. Stronger guided wave reflection, and correspondingly more pronounced absolute modulation of the major field components in the core region, is present for TM input. The TE-reflectance is not entirely zero, but on the same level as the 
conversion to TM. Our computations predict reflectance levels of $R_{\mathrm{TE} 0 \rightarrow \mathrm{TE} 0}=0.016, R_{\mathrm{TE} 0 \rightarrow \mathrm{TM} 0}=0.011$, $R_{\mathrm{TM} 0 \rightarrow \mathrm{TE} 0}=0.011, R_{\mathrm{TM} 0 \rightarrow \mathrm{TM} 0}=0.165$, where certainly the last digits must be suspected as being beyond the numerical level of accuracy.

Concerning field continuity, plots of suitable limits (not given) show that the e.m. components are as continuous, or suitably discontinuous, across the real and artificial interfaces as can be expected for the present piecewise expansion basis. For TE input, the largest electric field strengths are observed as peaks at the facet corners $\left(\left|E_{x}\right|\right)$ and on the end-interface of the facet $\left(\left|E_{z}\right|\right)$. When magnifying these regions, tiny oscillations ("Gibbs phenomena") become visible along the interfaces involved (cf. e.g. Ref. [19]). The limited spectral expansions on the bordering slices and layers can only be partly successful in approximating the non-smooth (divergent [19]) true fields at these points. Still, for sufficiently large expansion bases, and for the configurations tested, we never observed an unacceptably strong corruption of the field solutions by these artifacts.

With a view to existing BEP codes (also known by other names and acronyms) for scalar Helmholtz problems or mode analysis of channel waveguides, one might wonder whether not a description of the interior field in terms of a bidirectional expansion might be sufficient. As far as that can be assessed, a bidirectional simulation with our present code should be more or less equivalent to the method as proposed in Ref. [12]. Hence, we conclude the discussion of simulations for the facet configuration with a comparison between vQUEP and vBEP results. Figure 8 collects a number of respective graphs. vQUEP and vBEP simulations have been carried out for different computational windows of equivalent extent, with corresponding numbers of spectral terms (where roughly twice as many basis elements are used by the vQUEP as by the vBEP algorithm).

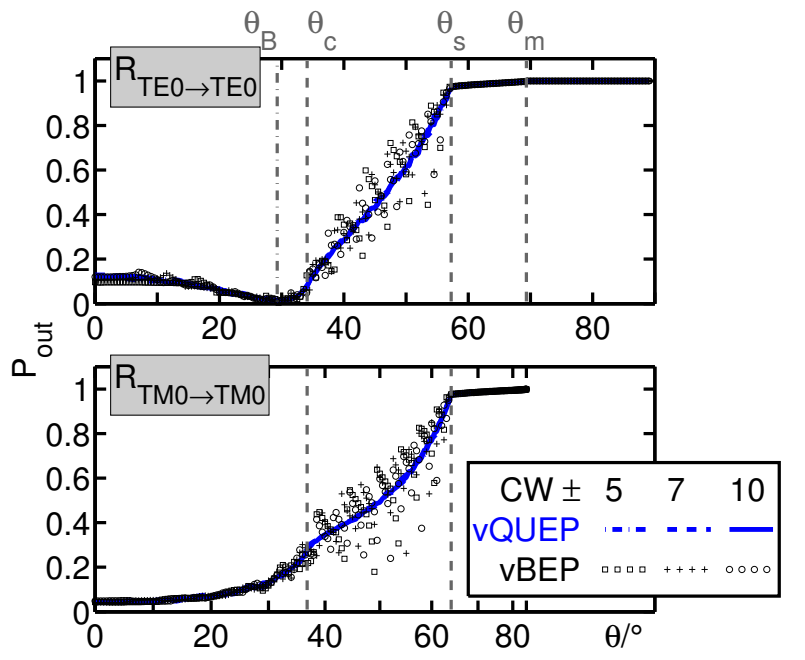

Figure 8: Modal reflectance versus angle of incidence, for $T E_{0}$ (top) and $T M_{0}$ excitation (bottom) of the facet of Figure 2. The curves correspond to VQUEP (lines) and $v B E P$ simulations (markers) for computational windows $(x, z) \in[-C W, C W] \times[-C W, C W](v Q U E P)$ and $x \in[-C W, C W](v B E P)$, respectively, with $C W$ as given in the legend, and for numbers of spectral terms $M_{x}=M_{z}$ (vQUEP) and $M_{x}(v B E P)$ of 90,150 , and 180 for the window extensions of $10 \mu \mathrm{m}, 14 \mu \mathrm{m}$, and $20 \mu \mathrm{m}$.

Already the vQUEP curves for the smallest window extension appear to be reasonably smooth and converged, on the entire range of angles, when compared to the data for the larger windows (the lines coincide on the scale of the plots). This cannot be observed for the vBEP results, where, at least in the intermediate range of angles with (partly) large radiative losses (cf. Figure 6(a)), even the curves for the widest vertical windows exhibit unacceptably large irregular oscillations. Note that the number of expansion terms $M_{x}$ has been increased roughly proportional with the computational window $\mathrm{CW}$, such that, for each $\mathrm{CW}$, the results appear to be reasonably converged with repect to $M_{x}$. Corresponding plots of the fields predicted by the vBEP solver at the angles in question show pronounced vertical standing waves that develop between the core and substrate/cover interface and the upper and lower artificial boundary. Hence these irregularities must be attributed to the unphysical truncation of the computational domain. This might be a reason why actually quite some part of Refs. [11, 12] is devoted to this phenomena, and why Ref. [12] has apparently not been followed up.

Note that the irregularities seem to be mostly restricted to the intermediate range of angles. One finds regular vBEP results for low angles of incidence, corresponding, in the limit $\theta=0$, to the standard scalar TE- and TM-Helmholtz problems [20, 21, 22], and for high angles of incidence, i.e. for configurations without radiative losses, relevant e.g. for BEP-based solvers for rectangular channel waveguides [23]. Obviously transparency of the upper and lower boundaries of the computational window, as implemented by the vQUEP scheme, is essential for simulations of the present configurations with their strong vertical radiative losses. One might thus anticipate that a corresponding vBEP technique equipped with other variants of transparent boundary conditions, e.g. suitably tuned perfectly matched layers [24], could work just as well as the vQUEP approach. 


\subsection{Lateral excitation of a strip waveguide}

Simulations of the structure of Figure 9, an example with slightly more complex cross section, emphasize the relation of the present type of computations with the modal analysis of 3-D channel waveguides. The facet of Section 4.1 is being extended by a single-mode strip waveguide of the same height, placed at some distance parallel to the facet interface.
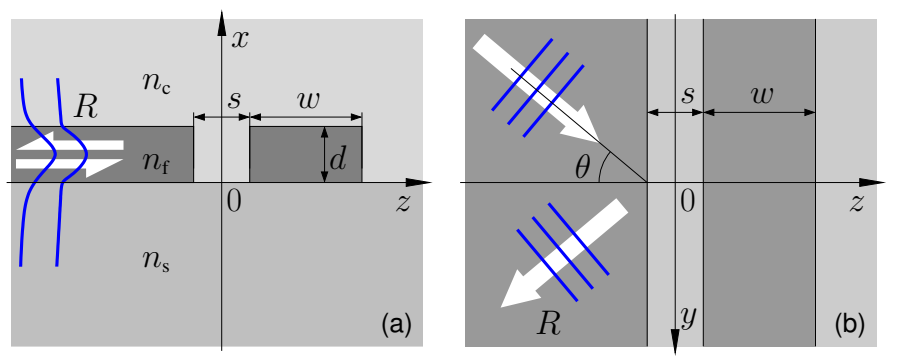

Figure 9: A strip waveguide running parallel to the former facet. Parameters are as given for Figure 2 $n_{\mathrm{c}}=1.0, n_{\mathrm{f}}=2.0, n_{\mathrm{s}}=1.5, d=0.5 \mu \mathrm{m}, \lambda=$ $1.55 \mu \mathrm{m}$, with additional values for the strip width $w=1.0 \mu \mathrm{m}$, and the gap distance $s=0.5 \mu \mathrm{m}$.

Figure 10 collects respective vQUEP results. The refractive index profiles that determine outgoing fields in all directions are the same as for the facet example, hence the discussion of critical angles $\theta_{\mathrm{c}}, \theta_{\mathrm{s}}, \theta_{\mathrm{m}}$, in Section 2.2 and the respective paragraphs of Section 4.1 apply here as well. There is also a region of small $R_{\mathrm{TE} 0 \rightarrow \mathrm{TE} 0}$ reflectance close to the quasi-Brewster angle $\theta_{\mathrm{B}}$ as introduced for the facet. When compared to Figure 6, the power transmission curves (a) are of quite similar form, with the exception of the features in the region just below $\theta=50^{\circ}$ (in: $\mathrm{TE}_{0}$ ) or just above that angle (in: $\mathrm{TM}_{0}$ ).
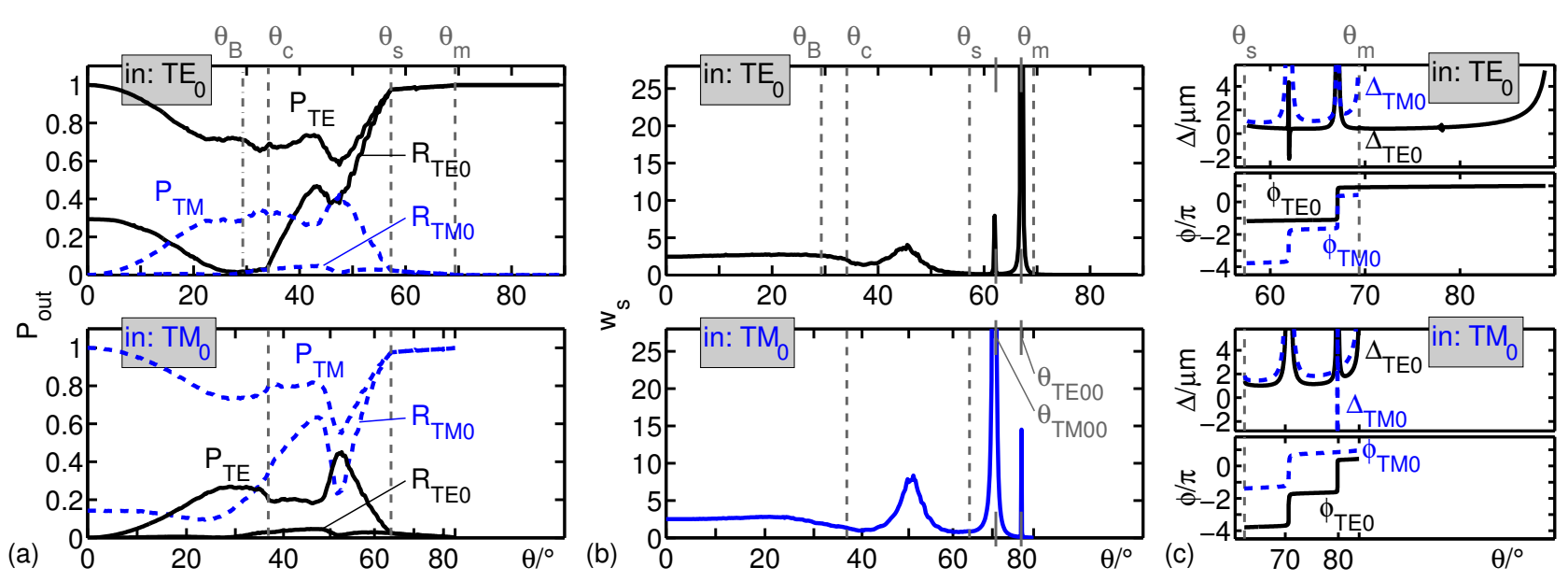

Figure 10: Oblique wave incidence on the strip-extended facet of Figure 9 , for varying angle of incidence $\theta$, and excitation by the fundamental TE- (top) and TM-mode (bottom). (a): Modal reflectance $R_{T E O}, R_{T M 0}$ and total relative polarized outgoing power $P_{T E}, P_{T M}$. (b): Electromagnetic energy density $w_{s}$, integrated over the strip core. (c): Modal phase gain upon reflection $\phi_{T E 0}, \phi_{T M 0}$, and respective beam displacement $\Delta_{T E 0}, \Delta_{T M 0}$.

Vectorial modal analysis [25] of the strip predicts two guided (hybrid) modes, one for each major polarization, with effective mode indices $N_{\mathrm{TE} 00}=1.641$ and $N_{\mathrm{TM} 00}=1.578$. In analogy to prism coupling setups for planar mode spectroscopy [26], for the present configuration, when scanning over the angle of incidence, one expects a response for a wavenumber $k_{y}$ (cf. Eq. (7)) that matches the propagation constants of the strip modes. Their effective indices can thus be translated to angles $\theta_{\mathrm{TE} 00}=66.98^{\circ}, \theta_{\mathrm{TM} 00}=62.25^{\circ}$, for TE excitation, and $\theta_{\mathrm{TE} 00}=79.49^{\circ}, \theta_{\mathrm{TM} 00}=70.99^{\circ}$, for TM-input. These are in the range between $\theta_{\mathrm{s}}$ and $\theta_{\mathrm{m}}$ of total guided wave reflection, where radiative losses are prohibited, but where the fundamental slab modes can both propagate.

Upon zooming in, the curves in Figure 10 (a) exhibit indeed tiny wiggles at the respective angles of incidence. The curves in (a) show the relative optical powers $R_{\mathrm{TE} 0}, R_{\mathrm{TM} 0}$ reflected into the two outgoing slab modes, with a major outgoing wave of the same polarization as the incoming mode, and a - tiny - contribution of orthogonal polarization. Apparently the mixture of polarizations changes with the angle of incidence around the resonances. Note, however, that these features appear on a level that is hardly better than our accuracy limit. As to be expected for the present non-attenuating media, the (numerically predicted) total reflected power $R_{\mathrm{TE} 0}+R_{\mathrm{TM} 0}$ remains constant (unity) for $\theta>\theta_{\mathrm{s}}$ on a reasonable level of scaling.

Better evidence of the resonant excitation of the strip modes is obtained by looking a the local optical intensity 
in the strip. The plots of the electromagnetic energy density, $x$ - $z$-integrated over the strip cross section, in Figure 10 (b) show sharp and pronounced peaks at positions $67.1^{\circ}, 62.0^{\circ}$ (in: $\mathrm{TE}_{0}$ ) and $79.8^{\circ}, 70.6^{\circ}$ (in: $\mathrm{TM}_{0}$ ) very near to the angles $\theta_{\mathrm{TE} 00}, \theta_{\mathrm{TM} 00}$. Figure 11 illustrates the fields for the major resonances, which indeed nicely resemble the modes profiles expected for the strip channel. Note that incoming and outgoing fields of unit power are present in the slab region next to the strip; these are hardly noticeable on the color-range of the panels.


Figure 11: Vectorial field profiles for the strip-extended facet of Figure 9, absolute values of the electric and magnetic components, for $T E_{0}$ incidence at $\theta=67.1^{\circ}$ (a), and for $T M_{0}$ incidence at $\theta=70.6^{\circ}$ (b). For the larger panels, the color-scales match the maxima of the individual field profiles, in order to provide some impression of also the shapes of small and minor components. For the smaller insets, the color scales have been adjusted such that plots in each row are comparable,

There is a small difference between the resonance positions observed in Figure 10 (b) and the angles as predicted by the mode analysis of the strip. If not fully attributable to the numerical uncertainty of either of the solvers, this might be due to the perturbation of the strip modes by the nearby slab core (coupling induced phase shifts, cf. e.g. Refs. [27, 28] 1 . The deviation appears to be slightly larger for the case of the $\mathrm{TM}_{00}$ resonance, with less confined fields, i.e. with stronger wave interaction.

In the absence of material losses, also the angular width of the peaks is being determined entirely by the interaction of waves associated with slab and strip. On the basis of the magnified plots, one roughly estimates full widths at half maxima of $0.01^{\circ}\left(\mathrm{TE}_{00}\right.$, in: $\left.\mathrm{TE}_{0}\right), 0.08^{\circ}\left(\mathrm{TM}_{00}\right.$, in: $\left.\mathrm{TE}_{0}\right), 0.02^{\circ}\left(\mathrm{TE}_{00}\right.$, in: $\left.\mathrm{TM}_{0}\right), 0.13^{\circ}$ $\left(\mathrm{TM}_{00}\right.$, in: $\left.\mathrm{TM}_{0}\right)$. Following e.g. Ref. [27, 28], a reasoning based on the "supermodes" associated with the "open system", comprising the actual cavity (the strip core) together with the outlet (the half-infinite slab), i.e. based on the (hybrid) leaky modes supported by the strip-slab configuration, can be applied. The lower quality of the $\mathrm{TM}_{00}$-related leaky mode then explains the broader angular features observed for excitation both by matching and by orthogonal polarization.

The curves in Figure 10 (b) show a third peak each, much broader and less pronounced, with maxima at roughly $\theta=46^{\circ}$ (in: $\mathrm{TE}_{0}$ ) and $\theta=52^{\circ}$ (in: $\mathrm{TM}_{0}$ ). The respective fields (not shown) have relative amplitudes in the strip core region of the same level as the fields in the slab region, with local field profiles that resemble the fields of horizontally $(z)$ first order strip modes. The deviation between the curves of Figure 6(a) and Figure 10 (a) in that angular region could thus relate to the excitation of leaky waves of higher order in the strip.

\footnotetext{
${ }^{1}$ Computations for gaps of widths $s=0.5 \mu \mathrm{m}, 0.4 \mu \mathrm{m}, 0.3 \mu \mathrm{m}$, and for $\mathrm{TE}_{0}$-excitation of the fundamental strip mode $\mathrm{TE}_{00}$ with $\theta_{\text {TE00 }}=66.98^{\circ}$, lead to peaks in the optical energy density at angles $\theta=67.08^{\circ}, 67.10^{\circ}, 67.14^{\circ}$.
} 
Part (c) of Figure 10 is concerned with the beam-shift properties of facet in the presence of the strip, restricted to the region where the strip modes play a role. When compared to Figure 6(c), the resonances show up as rapid (smooth) $2 \pi$-transitions, or, for the major reflectance's $\mathrm{TE}_{0} \rightarrow \mathrm{TE}_{0}$ and $\mathrm{TM}_{0} \rightarrow \mathrm{TM}_{0}$ at angles close to the strip mode of differing polarization, to sharp tiny wiggles in the phase curves. Both types of response lead to large, rapidly changing values (10) for the Goos-Hänchen-shift.

\subsection{Step discontinuity}

For purposes of comparison with (and assessment of) the scalar approach of Ref. [9], we briefly consider the transition between two slab waveguides of different thickness as introduced in the Figure 12 for oblique incidence of the $\mathrm{TE}_{0}$ mode of the thicker slab. Both slab regions (vertically symmetric) support guided fundamental TE- and TM modes, hence here outgoing transmitted guided waves appear.



(a)
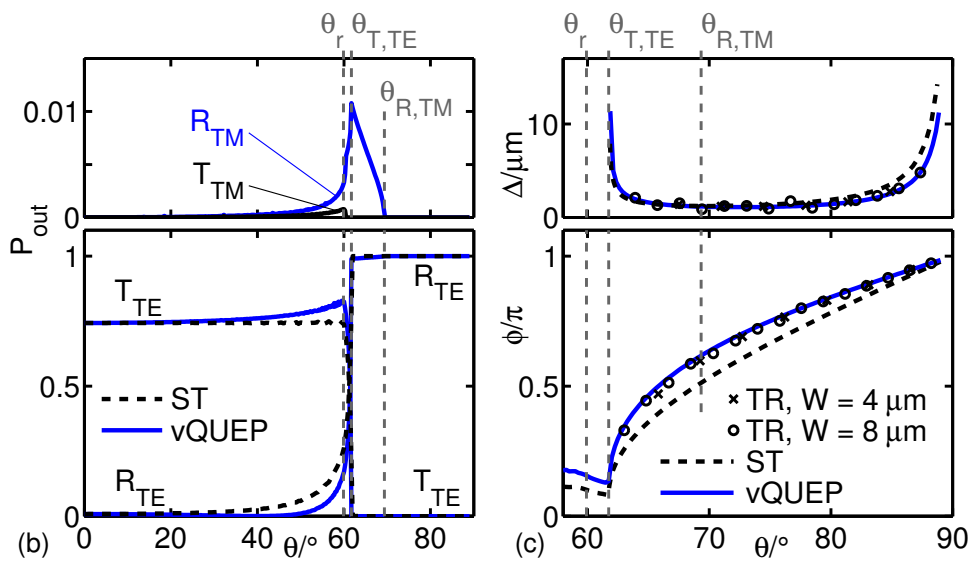

Figure 12: A straight step transition between two slab waveguides (a). Parameters [9]: refractive indices $n_{b}=1.4524$ (background), $n_{g}=2.0081$ (cores), slab thicknesses $d=0.160 \mu \mathrm{m}, r=0.040 \mu \mathrm{m}$, vacuum wavelength $\lambda=0.850 \mu \mathrm{m}$; excitation by the fundamental TE mode of the thicker slab. Results relate to the scalar theory (ST) of Ref. [9], to a transverse resonance (TR) model [9] based on semivectorial QTE modal solutions [29] for multimode rib waveguides of widths $W$, and to the present solver (vQUEP). Scans over the angle of incidence $\theta$, modal transmittance's $T_{T E}, T_{T M}$ and reflectance's $R_{T E}, R_{T M}(b)$, and phase $\phi$ of the major reflected wave and related beam displacement $\Delta$ (c). Critical angles $\theta_{r}=59.92^{\circ}, \theta_{T, T E}=61.75^{\circ}, \theta_{R, T M}=69.34^{\circ}$, and $\theta_{T, T M}=60.44^{\circ}$ (not shown) play a role.

Following the reasoning of Section 2.2, also here a couple of critical angles can be identified, based on the effective modal indices $N_{\text {in }}=N_{d, \mathrm{TE}}=1.678, N_{d, \mathrm{TM}}=1.571, N_{r, \mathrm{TE}}=1.479, N_{r, \mathrm{TM}}=1.460$ of the slab modes in the thick $(d)$ and thin regions $(r)$, respectively. Radiative losses are prohibited for incidence at angles $\theta>\theta_{\mathrm{r}}$ with $\sin \theta_{\mathrm{r}}=n_{\mathrm{b}} / N_{\mathrm{in}}$. Guided TE and TM waves cease to be transmitted for angles $\theta>\theta_{\mathrm{T}, \mathrm{TE}}$ or $\theta>\theta_{\mathrm{T}, \mathrm{TM}}$ with $\sin \theta_{\mathrm{T}, \mathrm{TE}}=N_{r, \mathrm{TE}} / N_{\mathrm{in}}$ and $\sin \theta_{\mathrm{T}, \mathrm{TM}}=N_{r, \mathrm{TM}} / N_{\mathrm{in}}$. Reflected guided TM waves are forbidden for $\theta>\theta_{\mathrm{R}, \mathrm{TM}}$ with $\sin \theta_{\mathrm{R}, \mathrm{TM}}=N_{d, \mathrm{TM}} / N_{\mathrm{in}}$. Respective singular features can be observed for the curves of Figure 12 .

According to the present analysis, the relative conversion to guided outgoing TM modes remains below $1 \%$ for all angles of incidence. The scalar analysis (scalar theory ST, based on an angle-dependent effective permittivity profile, scalar TE-QUEP solver) thus appears to be mostly adequate, with more pronounced deviations for angles between $40^{\circ}$ and $\theta_{\mathrm{T}, \mathrm{TE}}$. Emphasis in Ref. [9], however, was on the regime of full TE reflectance, and in particular on the phase properties of the reflectance. One observes that the largest (still small) levels of conversion to guided TM waves appear in the region immediately beyond $\theta_{\mathrm{T}, \mathrm{TE}}$.

Concerning the phase $\phi$ of the reflected TE wave, and the related Goos-Hänchen-shift $\Delta$, part (c) of Figure 12 compares the results from Ref. [9] with our present vQUEP solver. Accepting that as benchmark, both methods from [9] produce adequate approximations, with the TR results $(\phi)$ being slightly closer to the reference. That data is based on an argument of transverse resonance, based on the semivectorial modal analysis [29] of wide multimode rib waveguides, which are formed by two mirrored step discontinuities at some distance $W$. Polarization issues at the rib sidewalls (i.e. at the step transition), are at least partially taken into account by the TR analysis. Thus there appears to be some not fully negligible influence of the polarization effects on the phase of the reflected wave. 


\section{Concluding remarks}

The vectorial variant of a scheme for quadridirectional eigenmode propagation, intended for problems of oblique quasi-guided wave incidence on rectangular dielectric structures, that are constant along one coordinate axis, has been discussed. Just as the scalar QUEP version [13], upon which the present solver is built, the vQUEP scheme realizes transparent boundary conditions on a rectangular computational window, using real basis functions only (the principal mode shapes $\psi$ are solutions of Sturm-Liouville eigenvalue problems with real eigenvalues $\beta^{2}$ ). The solver provides convenient access to both the modal transmission coefficients as well as to the full vectorial optical electromagnetic field.

Beyond the actual results for the specific configurations, the numerical examples provide some means of assessment for the vQUEP solver, if only via internal checks. These include consistency (power conservation, reciprocity), and in particular the inspection of the generated fields. Basically, the vectorial equations underneath the present simulations also govern the eigenmodes of 3-D channel waveguides. Consequently, as illustrated by the example of the laterally coupled strip, one sees similar effects, e.g. of field hybridization / polarization conversion, or of field divergence at permittivity corners [19], in both types of problems.

The present implementation permits, in principle, the investigation of structures with quite arbitrary rectangular cross sections, with multiples of horizontal and vertical interfaces. This might include configurations with also vertically outgoing slabs, such as e.g. oblique wave incidence on a $90^{\circ} \mathrm{kink} /$ corner in a slab waveguide.

\section{Acknowledgments}

The author likes to thank the members of the TET group, and in particular J. Förstner, for the hospitality experienced during the stay at the University of Paderborn.

\section{References}

[1] P. K. Tien. Integrated optics and new wave phenomena in optical waveguides. Reviews of Modern Physics, 49(2):361-419, 1977.

[2] R. Ulrich and R. J. Martin. Geometrical optics in thin film light guides. Applied Optics, 10(9):2077-2085, 1971.

[3] G. C. Righini, V. Russo, S. Sottini, and G. Toraldo di Francia. Geodesic lenses for guided optical waves. Applied Optics, 12(7):1477-1481, 1973.

[4] F. Zernike. Luneburg lens for optical waveguide use. Optics Communications, 12(4):379-381, 1974.

[5] S. Misawa, M. Aoki, S. Fujita, A. Takaura, T. Kihara, K. Yokomori, and H. Funato. Focusing waveguide mirror with a tapered edge. Applied Optics, 33(16):3365-3370, 1994.

[6] C.-C. Tseng, W.-T. Tsang, and S. Wang. A thin-film prism as a beam separator for multimode guided waves in integrated optics. Optics Communications, 13(3):342-346, 1975.

[7] G. C. Righini and G. Molesini. Design of optical-waveguide homogeneous refracting lenses. Applied Optics, 27(20):4193-4199, 1988.

[8] F. Çivitci, M. Hammer, and H. J. W. M. Hoekstra. Design of a prism spectrometer based on adiabatically connected waveguiding slabs, Journal of Lightwave Technology (submitted for publication, 2013).

[9] F. Çivitci, M. Hammer, and H. J. W. M. Hoekstra. Semi-guided plane wave reflection by thin-film transitions for angled incidence. Optical and Quantum Electronics, 46(3):477-490, 2014.

[10] C. Vassallo. Optical Waveguide Concepts. Elsevier, Amsterdam, 1991.

[11] T. P. Shen, R. F. Wallis, A. A. Maradudin, and G. I. Stegeman. Fresnel-like behavior of guided waves. Journal of the Optical Society of America A, 4(11):2120-2132, 1987.

[12] W. Biehlig and U. Langbein. Three-dimensional step discontinuities in planar waveguides: Angular-spectrum representation of guided wavefields and generalized matrix-operator formalism. Optical and Quantum Electronics, 22(4):319-333, 1990.

[13] M. Hammer. Quadridirectional eigenmode expansion scheme for 2-D modeling of wave propagation in integrated optics. Optics Communications, 235(4-6):285-303, 2004. 
[14] A. W. Snyder and J. D. Love. Optical Waveguide Theory. Chapman and Hall, London, New York, 1983.

[15] M. Lohmeyer and R. Stoffer. Integrated optical cross strip polarizer concept. Optical and Quantum Electronics, 33(4/5):413-431, 2001.

[16] H. K. V. Lotsch. Reflection and refraction of a beam of light at a plane interface. Journal of the Optical Society of America, 58(4):551-561, 1968.

[17] Chun-Fang Li. Negative lateral shift of a light beam transmitted through a dielectric slab and interaction of boundary effects. Physical Review Letters, 91:133903, 2003.

[18] M. Hammer. METRIC - Mode expansion tools for 2D rectangular integrated optical circuits. http://metric.computational-photonics.eu/.

[19] A. S. Sudbø. Why are accurate computations of mode fields in rectangular dielectric waveguides difficult? Journal of Lightwave Technology, 10(4):418-419, 1992.

[20] W. Biehlig, K. Hehl, U. Langbein, and F. Lederer. Light propagation in a planar dielectric slab waveguide with step discontinuities, Part 1: Operator formalism. Optical and Quantum Electronics, 18(3):219-228, 1986.

[21] W. Biehlig. Light propagation in a planar dielectric slab waveguide with step discontinuities, Part 2: Numerical analysis of TE-polarized fields. Optical and Quantum Electronics, 18(3):229-238, 1986.

[22] W. Biehlig and Ch. Wächter. Light propagation in a planar dielectric slab waveguide with step discontinuities, Part 3: TM-polarized fields. Optical and Quantum Electronics, 18(3):239-246, 1986.

[23] A. S. Sudbø. Film mode matching: a versatile numerical method for vector mode fields calculations in dielectric waveguides. Pure and Applied Optics, 2:211-233, 1993.

[24] P. Bienstmann and R. Baets. Advanced boundary conditions for eigenmode expansion models. Optical and Quantum Electronics, 34(5/6):523-540, 2002.

[25] M. Lohmeyer. Vectorial wave-matching mode analysis of integrated optical waveguides. Optical and Quantum Electronics, 30:385-396, 1998.

[26] R. G. Hunsperger. Integrated Optics: Theory and Technology, Fourth Edition. Springer Verlag, Berlin, 1995.

[27] M. A. Popović, C. Manolatou, and M. R. Watts. Coupling-induced resonance frequency shifts in coupled dielectric multi-cavity filters. Optics Express, 14(3):1208-1222, 2006.

[28] E.F. Franchimon, K.R. Hiremath, R. Stoffer, and M. Hammer. Interaction of whispering gallery modes in integrated optical micro-ring or -disk circuits: Hybrid CMT model. Journal of the Optical Society of America B, 30(4):10481057, 2013.

[29] M. Lohmeyer. Wave-matching method for mode analysis of dielectric waveguides. Optical and Quantum Electronics, 29:907-922, 1997. 